\title{
Sensibilidade do carrapato Boophilus microplus a solventes $^{1}$
}

\author{
Sensibility of Boophilus microplus tick to solvents
}

\section{Ana Carolina de Souza Chagas ${ }^{2}$ Romário Cerqueira Leite ${ }^{3}$ John Furlong ${ }^{4}$ Hélio Teixeira Prates ${ }^{5}$ Wanderley Mascarenhas Passos ${ }^{6}$}

\section{RESUMO}

\begin{abstract}
Os experimentos envolvendo o uso de acaricidas sintéticos ou naturais, geralmente necessitam da utilização de um solvente. Com a finalidade de verificar a sensibilidade do carrapato bovino Boophilus microplus a diferentes solventes, larvas e fêmeas ingurgitadas deste ectoparasito foram expostas a sete solventes em cinco diferentes concentrações, na ausência e presença de azeite de oliva. Os resultados mostraram que a utilização do azeite de oliva não produz resultados diferentes estatisticamente em testes de larvas com papel impregnado, fato não verificado em testes de imersão de adultos com compostos hidrofílicos. A mortalidade média causada pelos solventes foi menor nos testes com papel impregnado, aumentando nos testes de imersão de larvas e de adultos. Solventes de baixo peso molecular e pouca viscosidade como o álcool metílico e o álcool etílico, não interferiram na mortalidade média em testes biológicos de B. microplus, principalmente em concentrações inferiores a $76 \%$.
\end{abstract}

Palavras-chave: Boophilus microplus, solventes, testes biológicos, acaricida.

\section{ABSTRACT}

Experiments carried out with synthetic or natural acaricides usually use a solvent. To investigate the sensitivity of Boophilus microplus cattle tick to different solvents, larvae and engorged female were subjected to seven solvents in five different concentrations. It was done in the presence and absence of olive oil. The results showed that the utilization of olive oil doesn't produce different statistical results in impregnated paper larvae test. It did not happen in adults immersion test with hydrophilic compounds. The mean mortality caused by solvents was small in impregnated paper larvae test, increasing in immersion tests of larvae and adults. Solvents with low molecular weight and viscosity like ethyl alcohol and methyl alcohol did not cause interference in the mortality of $\boldsymbol{B}$. microplus in biological tests, mainly in concentrations below $76 \%$.

Key words: Boophilus microplus, solvents, biological tests, acaricide.

\section{INTRODUÇÃO}

O carrapato Boophilus microplus (Canestrini, 1887) (Acari: Ixodidae) parasita principalmente os bovinos por ser extremamente específico, mas pode esporadicamente parasitar outros animais, tais como eqüinos e ovinos (GONZALES, 1974). Os carrapaticidas têm sido o principal meio de controle do B. microplus, entretanto a capacidade desse parasita tornar-se resistente, tem prejudicado muito a aplicação dos carrapaticidas. Quando a resistência se instala, o

${ }^{1}$ Parte da tese de doutorado em Ciência Animal da Universidade Federal de Minas Gerais (UFMG) do primeiro autor.

${ }^{2}$ Doutorando da UFMG, Avenida Governador Valadares, 1002, Manoel Honório, 36051-550, Juiz de Fora, MG. Fone: (32)3226-

5330. E-mail: carolina@cnpc.embrapa.br. Autor para correspondência.

${ }^{3}$ Professor da Escola de Veterinária da UFMG.

${ }^{4}$ Pesquisador da EMBRAPA Gado de Leite;

${ }^{5}$ Pesquisador da EMBRAPA Milho e Sorgo;

${ }^{6}$ Professor do Curso de Controle de Vetores da Fiocruz e Pesquisador do PSI/Embrapa/Universidade Federal Rural do Rio de Janeiro: Recebido para publicação 04.07.01 Aprovado em 13.03.02 
produtor freqüentemente aumenta a dose do pesticida ou a freqüência das aplicações (THULLNER, 1997). O uso indiscriminado dos defensivos químicos afeta o ambiente, os animais e as pessoas, mas com o uso de produtos de origem natural, o desequilíbrio ecológico e a contaminação ambiental poderiam ser minimizados (HERNÁNDEZ et al., 1987).

Existem vários tipos de solventes que fazem parte das formulações carrapaticidas ou que são utilizados em experimentos com extratos vegetais. Eles têm a principal finalidade de solubilizar o princípio ativo, proporcionando a distribuição homogênea do mesmo por toda a cutícula do artrópodo, ampliando sua área de ação. São poucos os trabalhos comparativos com relação à interferência dos diferentes solventes no resultado final de um experimento, ou ainda se diferentes estádios de um parasita reagem diferentemente na presença de um mesmo solvente. SHAW (1966) demonstra que a $\mathrm{DL}_{50}$ de "dioxathion" (fosforado) diluído em xilol é de $0,00015 \%$, enquanto que em nafta aromática pesada passa a ser de $0,00035 \%$. Segundo BEADLES et al. (1973), sempre é importante distinguir entre o efeito do produto como um todo e dos demais componentes da fórmula sem o princípio ativo, incluindo aí os solventes. Este é o procedimento que tem sido preconizado pela Organização Mundial para a Alimentação e a Agricultura das Nações Unidas (FAO PLANT PROTECTION BULLETIN, 1971).

Dentre os poucos trabalhos que estudaram a ação de solventes, podem-se citar HADAWAY \& BARLOW (1958) em Aedes aegypti e Musca domestica; REED et al. (1968) nos ácaros Phyllocoptruta oleivora e Aculops pelekassi; THIELE \& HARRISON (1968) no ácaro Tetranychus urticae; BEADLES et al. (1973) em Anocentor nitens. Não foram encontrados trabalhos comparativos da ação dos solventes em $\boldsymbol{B}$. microplus.

Neste trabalho, procurou-se observar a sensibilidade de larvas e fêmeas ingurgitadas de $\boldsymbol{B}$. microplus frente a diferentes solventes, comparandose os diferentes métodos empregados.

\section{MATERIAL EMÉTODOS}

\section{1) Sensibilidade larval em papeis impregnados}

Foram utilizadas duas repetições para cada solvente de pureza analítica (PA) adquiridos comercialmente (todos do Laboratório VETEC Química Fina Ltda.): álcool etílico, álcool metílico, acetona, acetato de etila, dimetilsulfóxido (DMSO), uma mistura contendo triton X-100 ( $0,05 \%)$, água $(0,6 \%)$ e álcool etílico $(0,3 \%)$, e ainda o xilol, além do controle contendo água. A maioria destas substâncias é considerada como um solvente adequado e de fácil aquisição. Os solventes foram testados na concentração de $100 \%$ e estipulouse que aqueles que provocassem mortalidade superior a $5 \%$ seriam também testados a 75,50 e $25 \%$. O xilol também foi testado a $5 \%$ em função da elevada mortalidade encontrada. $\mathrm{O}$ acetato de etila e o xilol, por serem pouco solúveis em água, precisaram ser solubilizados em álcool metílico em todos os testes deste trabalho, após detectar-se sua baixa toxicidade em B. microplus.

Para a realização do teste utilizou-se a técnica adaptada por LEITE (1988): aproximadamente 100 larvas foram colocadas entre 2 pedaços de papel-filtro $(2 \times 2 \mathrm{~cm})$ impregnados por cada um dos solventes $(0,4 \mathrm{~mL}$ para cada repetição). Este "sanduíche" foi colocado em um envelope de papel-filtro $(6 \times 6 \mathrm{~cm})$ e foi vedado por pregadores plásticos, segundo FAO PLANT PROTECTION BULLETIN (1971). Os envelopes foram colocados em estufa climatizada $\left( \pm 27^{\circ} \mathrm{C}\right.$ e UR $\left.>80 \%\right)$ e o registro de larvas vivas e mortas foi realizado após 24 h. Os solventes foram testados na ausência e na presença de azeite de oliva (uma gota sobre as larvas no papel impregnado).

Para a obtenção das larvas de B. microplus, utilizaram-se fêmeas ingurgitadas de uma cepa sensível (Porto Alegre, RS) provenientes de colônia da Estação Experimental de Coronel Pacheco, Embrapa Gado

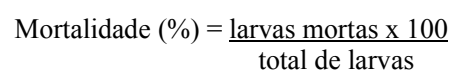

Mortalidade

média $(\%)=\frac{\text { mortalidade da repetição } 1+\text { mortalidade da repetição } 2}{2}$

de Leite, Zona da Mata de Minas Gerais. Utilizaram-se somente larvas com 14 a 21 dias de idade.

Para o cálculo da mortalidade, foi utilizada a seguinte fórmula:

$$
\begin{aligned}
& \text { Mortalidade }(\%)=\text { larvas mortas } \times 100 \\
& \text { total de larvas }
\end{aligned}
$$

Mortalidade

média $(\%)=$ mortalidade da repetição $1+$ mortalidade da repetição 2

2

Não foram incluídos testes que produziram resultados com mortalidade no grupo controle superior a $10 \%$. Nos testes com mortalidade no grupo controle entre 5 e $10 \%$, cada mortalidade média foi corrigida aplicando-se a fórmula de ABBOTT (1925). Não foram feitas correções quando a mortalidade do controle foi entre 0 e $5 \%$ e a mortalidade do teste foi de 0 ou $100 \%$. 


\section{2) Sensibilidade larval em testes de imersão}

Utilizou-se a técnica desenvolvida por SHAW (1966), que consistiu basicamente na imersão de aproximadamente 300 larvas de carrapato por 10 minutos nos mesmos solventes e nas mesmas concentrações supracitadas. Após a exposição, a larvas foram colocadas em papéis- filtro para secagem e aproximadamente 100 exemplares vivos foram transferidos para envelopes de papéis-filtro secos e posteriormente acondicionados em estufa climatizada. O registro de larvas vivas e mortas foi realizado após 24h. Optou-se por não se avaliar a ação do azeite neste teste, pelo fato do tempo de imersão ser maior e o contato com os solventes mais intenso que no teste com papel de filtro impregnado. O teste foi realizado com duas repetições.

\section{3) Sensibilidade das fêmeas ingurgitadas em testes de imersão}

Seguindo-se metodologia semelhante à descrita por DRUMMOND et al. (1973), grupos de 10 fêmeas foram pesadas e imersas por cinco minutos nos mesmos solventes e nas mesmas concentrações. Elas foram acondicionadas em estufa climatizada e tiveram seus ovos pesados e a eclodibilidade estimada visualmente, possuindo esse teste duas repetições. $\mathrm{O}$ azeite de oliva também foi testado, colocando-se $0,5 \mathrm{~mL}$ do mesmo em $20 \mathrm{~mL}$ dos solventes. A mortalidade das fêmeas foi calculada segundo a fórmula de porcentagem de controle ou de eficácia, descrita por DRUMMOND et al. (1973).

Os resultados desse trabalho foram submetidos ao Procedimento Genreal Linear Model (SAS, 1990), que usa princípios de quadrados mínimos para ajustar modelos lineares e o contraste entre médias foi feito pelo Teste Student Newman Keuls. de $100 \%$ e por isso foi testado também a $5 \%$, demonstrando mortalidade de $1 \%$ em $24 \mathrm{~h}$ e de $2,4 \%$ em $48 \mathrm{~h}$ (sem azeite) e de $4,2 \%$ em $24 \mathrm{~h}$ e de $39,4 \%$ em $48 \mathrm{~h}$ (com azeite). Os solventes com azeite deixados em contato com as larvas durante $48 \mathrm{~h}$, provocaram maior mortalidade que na ausência do azeite com $24 \mathrm{~h}$, mas tais diferenças não foram estatisticamente significativas $(\mathrm{p}<0,05)$.

No teste de imersão de larvas, o álcool etílico a $100 \%$ apresentou mortalidade média inferior a 5\%, ocorrendo o mesmo com o álcool metílico a 75\%, com a acetona a $50 \%$ e com a mistura de triton a $25 \%$. O DMSO e o acetato de etila apresentaram elevadas taxas de mortalidade mesmo a $25 \%$, o que também ocorreu com o xilol a $5 \%$ (Tabela 1 ).

No teste de imersão de fêmeas ingurgitadas, o álcool metílico, o álcool etílico e a acetona demonstraram pouca toxicidade na ausência do azeite, mas sua presença aumentou esta toxicidade significativamente. A mortalidade média causada pelos solventes na presença do azeite foi de $61,2 \%$ e na ausência de $49 \%$, sendo estes valores diferentes estatisticamente $(p<0,05)$. O crescimento da concentração do acetato de etila e da mistura com triton (com e sem azeite) não foi acompanhado por um crescimento linear da mortalidade média (Tabela 2).

A imersão de fêmeas causou mortalidade média geral de $55,1 \%$, a imersão de larvas de $40,5 \%$ e a metodologia de papéis impregnados para larvas causou mortalidade de $30,4 \%$ (Tabela 3 ), sendo estes valores diferentes estatisticamente $(\mathrm{p}<0,05)$. Os dados transformados para logaritmo indicaram que os solventes tiveram um padrão de penetração semelhante para os dois estádios, pois o álcool metílico, o álcool etílico e a acetona, causaram maior mortalidade com o aumento da concentração. Os demais sempre causaram grande mortalidade, principalmente os testes de imersão de larvas e de fêmeas ingurgitadas.

\section{RESULTADOS}

No teste com papel impregnado, as larvas de $\boldsymbol{B}$. microplus não sofreram ação letal superior a $5 \%$ diante do álcool metílico, álcool etílico, acetona, acetato de etila e mistura com triton, na concentração de $100 \%$. No entanto, o DMSO a $25 \%$ causou mortalidade de $22,5 \%$ em 24 h e de $93 \%$ em $48 \mathrm{~h}$ (sem azeite) e de $73 \%$ em $24 \mathrm{~h}$ e de $100 \%$ em $48 \mathrm{~h}$ (com azeite). O xilol causou mortalidade média
Tabela 1 - Mortalidade média (\%) de lavras de Boophilus microplus submetidas ao teste de imersão com solventes

\begin{tabular}{cccccc}
\hline Solventes & & \multicolumn{3}{c}{ concentrações } & \\
& $100 \%$ & $75 \%$ & $50 \%$ & $25 \%$ & $5 \%$ \\
\hline Controle & 0,5 & & & $*$ & $*$ \\
Álcool metílico & 15,4 & 0 & $*$ & $*$ & $*$ \\
Álcool etílico & 3,9 & $*$ & $*$ & $*$ & $*$ \\
Acetona & 8,9 & 6,7 & 0 & 45 & $* *$ \\
DMSO & 93,5 & 100 & 67 & 41,3 & $* *$ \\
Acetato de etila & 100 & 100 & 100 & 2,1 & $*$ \\
Mistura de triton & 36,4 & 77,5 & 7 & 93 & 20,2 \\
Xilol & 100 & 100 & 100 & & \\
\hline
\end{tabular}

* Quando a mortalidade era $\leq$ a 5\% não eram feitos os demais testes.

** Teste não realizado. 


\begin{tabular}{|c|c|c|c|c|c|c|c|c|c|c|c|c|c|c|}
\hline \multirow[b]{2}{*}{ Conc. } & \multicolumn{7}{|c|}{ sem azeite } & \multicolumn{7}{|c|}{ com azeite } \\
\hline & $\mathrm{M}$ & $\mathrm{E}$ & $\mathrm{A}$ & $\mathrm{D}$ & $\mathrm{AE}$ & $\mathrm{T}$ & $X$ & $\mathrm{M}$ & $\mathrm{E}$ & $\mathrm{A}$ & $\mathrm{D}$ & $\mathrm{AE}$ & $\mathrm{T}$ & $\mathrm{X}$ \\
\hline $100 \%$ & 15 & 56 & 100 & 89 & 100 & 70 & 100 & 79 & 83 & 100 & 83 & 100 & 69 & 100 \\
\hline $75 \%$ & 0 & 0 & 10 & 55 & 88 & 72 & 100 & 36 & 19 & 83 & 81 & 33 & 90 & 100 \\
\hline $50 \%$ & 0 & 0 & 2 & 47 & 59 & 61 & 100 & 18 & 17 & 8 & 80 & 77 & 30 & 100 \\
\hline $25 \%$ & 0 & 0 & 0 & 24 & 78 & 53 & 100 & 8 & 22 & 18 & 58 & 40 & 90 & 100 \\
\hline $5 \%$ & $*$ & $*$ & $*$ & $*$ & $*$ & $*$ & 92 & $*$ & $*$ & $*$ & $*$ & $*$ & $*$ & 89 \\
\hline
\end{tabular}

*Teste não realizado.

\section{DISCUSSÃO}

O teste de imersão de larvas demonstra possibilitar um contato maior com o solvente do que o teste com papel impregnado, permitindo a entrada do produto pela cutícula do carrapato mais eficientemente. Observa-se também que a presença do azeite de oliva no teste de papel impregnado não provoca diferença estatística significativa com relação aos resultados obtidos na sua ausência. No teste de imersão de fêmeas ingurgitadas, a presença do azeite aumentou a mortalidade média causada pelos solventes hidrofílicos. De uma maneira geral, as fêmeas se mostraram mais sensíveis aos solventes do que as larvas, embora estas ficassem 5 minutos a mais em contato com os mesmos no teste de imersão. Segundo ODHIAMBO (1982), no início do processo de alimentação a espessura da cutícula aumenta, mas depois ela é esticada e se torna de espessura igual ou parecida à da larva. Durante a alimentação nos ixodídeos, a síntese cuticular aumenta bastante, mas quando a cutícula está esticada ao final deste processo, ela volta praticamente à sua espessura original (GEROLT, 1970). Pouca informação foi encontrada a respeito desse processo especificamente em $\boldsymbol{B}$. microplus, mas tudo indica que a espessura da cutícula não justificaria a maior sensibilidade das fêmeas aos solventes.

Tabela 3 - Comparação de mortalidade média geral (\%) dos solventes sobre Boophilus microplus nos três métodos utilizados.

\begin{tabular}{cccc}
\hline Solventes & Contato larva & Imersão larva & Imersão fềmea \\
\hline Álcool metílico & $2,3 \mathrm{a}$ & $3,9 \mathrm{a}$ & $19,5 \mathrm{a}$ \\
Álcool etílico & $3,5 \mathrm{a}$ & $1,0 \mathrm{a}$ & $24,6 \mathrm{a}$ \\
Acetona & $2,2 \mathrm{a}$ & $3,9 \mathrm{a}$ & $40,1 \mathrm{~b}$ \\
DMSO & $100 \mathrm{~b}$ & $76,4 \mathrm{~b}$ & $64,6 \mathrm{c}$ \\
Acetato de etila & $2,7 \mathrm{a}$ & $85,3 \mathrm{~b}$ & $71,9 \mathrm{c}$ \\
Triton & $2,4 \mathrm{a}$ & $30,7 \mathrm{c}$ & $66,9 \mathrm{c}$ \\
Xilol & $100 \mathrm{~b}$ & $82,6 \mathrm{~b}$ & $98,1 \mathrm{~d}$ \\
Média & 30,4 & 40,5 & 55,1 \\
\hline
\end{tabular}

*Média com a mesma letra, na mesma coluna, não são significativamente diferentes $(\mathrm{p}<0,005)$
Acreditase que a diferença de composição da cutícula entre larvas e fêmeas ingurgitadas é que possa justificar os resultados obtidos. De acordocomBALASHOV (1972), a cutícula dos carrapatos é formada pela camada externa, epicutícula (composta externamente por ceras e internamente por proteínas) e também pela camada interna, procutícula (quitina e proteína). Segundo ODHIAMBO (1982), a camada de ceras ou de lipídeos é vista em $\boldsymbol{B}$. microplus somente a partir da ecdise na ninfa e, em maior quantidade, no adulto. Portanto, nas fêmeas ingurgitadas, os solventes precisam dissolver primeiro a camada de ceras da epicutícula, para só então passar para as camadas mais polares da cutícula, constituídas de proteínas hidrossolúveis. Quanto mais um produto químico for lipossolúvel, maior será a facilidade inicial que encontrará para penetrar a cutícula (ODHIAMBO, 1982). Este autor comenta que a produção de lipídeos aumenta enquanto a fêmea se alimenta. Quando ela cai do hospedeiro, essa camada de cera protege a grande quantidade de água que existe em seu interior e além disso, seu principal componente é o colesterol, que funciona como um feromônio sexual de cópula. Em B. microplus a quantidade de lipídeos na camada externa passa de $22 \mu \mathrm{g}$ para $63 \mu \mathrm{g}$ durante a alimentação (SONENSHINE, 1991). A maior toxicidade revelada pelo álcool metílico, álcool etílico e acetona na presença do azeite, indica a importância deste agente carreador na potencialização destes solventes com fêmeas ingurgitadas. Quando a camada de ceras é destruída nas fêmeas ingurgitadas, a grande perda de água para o meio é inevitável.

Segundo PASSOS (1994), a celulose do papel diminui a toxicidade de alguns defensivos químicos, capturando o princípio ativo e retendo-o. Talvez este fato também tenha colaborado na menor mortalidade detectada nos dois testes realizados com larvas em relação às fêmeas, já que ambos utilizaram papel de filtro.

HADAWAY \& BARLOW (1958) afirmam que o aumento da ação do solvente sobre a cutícula do inseto, está relacionado ao aumento do peso molecular, ponto de ebulição e viscosidade do mesmo. No presente trabalho os solventes mais viscosos 
Tabela 4 - Solventes de pureza analítica (PA) testados contra Boophilus microplus, sua fórmula química e peso molecular.

\begin{tabular}{ccc}
\hline Solventes & Fórmula & Peso molecular \\
\hline Álcool metílico & $\mathrm{CH}_{3} \mathrm{OH}$ & 32,04 \\
Álcool etílico & $\mathrm{C}_{2} \mathrm{H}_{5} \mathrm{OH}$ & 46,07 \\
Acetona & $\mathrm{C}_{3} \mathrm{H}_{6} \mathrm{O}$ & 58,08 \\
DMSO & $\mathrm{C}_{2} \mathrm{H}_{6} \mathrm{SO}$ & 48,13 \\
Acetato de etila & $\mathrm{C}_{4} \mathrm{H}_{8} \mathrm{O}_{2}$ & 88,11 \\
Triton* & $\mathrm{C}_{34} \mathrm{H}_{62} \mathrm{O}_{11}$ & 646,86 \\
Xilol & $\mathrm{C}_{8} \mathrm{H}_{10}$ & 106,16 \\
\hline
\end{tabular}

*Concentração máxima de 0,05\%.

e/ou de maior peso molecular, provocaram maior mortalidade nos testes de imersão (Tabela 4). Os mesmos autores afirmam que o aumento da viscosidade pode não estar relacionado com o aumento linear da concentração letal e isto foi observado com o acetato de etila e com a mistura com triton. BEADLES et al. (1973) observaram que os solventes que possuem mais de 8 carbonos em sua estrutura impedem a oviposição. Tal fato ocorreu no presente trabalho com o xilol e embora o triton tenha 34 carbonos, o mesmo foi testado a uma concentração de $0,05 \%$ para baixo, o que deve ter minimizado sua ação sobre $\boldsymbol{B}$. microplus.

HARRISON (1961) sugere a utilização de querosene com no máximo $10 \%$ de xilol, em experimentos com aplicação tópica. No presente trabalho observou-se que a utilização do xilol a $10 \%$ causaria alta mortalidade nos métodos utilizados. Segundo THIELE \& HARRISON (1968), a acetona é muito utilizada em aplicação tópica, por evaporar rapidamente deixando o inseticida depositado de maneira uniforme sobre a cutícula do inseto. O Dimetilsulfóxido é considerado um excelente permeabilizador de membranas, permitindo a penetração do produto a ser testado de maneira rápida. No entanto, o fato de causar alta mortalidade em B. microplus prejudica seu uso. DRUMMOND et al. (1973) indicam a utilização de 25 partes de inseticida, 10 partes de Triton X-100 e 65 partes de xileno na produção de concentrados emulsionáveis. Todavia o Triton nessa concentração (10\%) causaria mortalidade média maior que as detectadas no presente trabalho, onde foi testado a uma concentração máxima de $0,05 \%$.

Os testes de extratos com potencial inseticida têm utilizado o álcool etílico, o álcool metílico e a acetona (CONSOLI et al., 1988; KARR \& COATS, 1988; SUJUTHA et al., 1988). Especificamente com $\boldsymbol{B}$. microplus, CHUNGSAMARNYART \& JIWAJINDA (1992) e CHUNGSAMARNYART \& JANSAWAN (1996) utilizaram álcool etílico a 95\% na diluição de óleos extraídos de plantas, na detecção de sua ação acaricida. WILLIAMS (1993) e PORTER et al. (1995) utilizaram acetona para testes de aplicação tópica de extratos vegetais sobre fêmeas de B. microplus. Esses trabalhos indicaram a pouca toxicidade desses solventes hidrofílicos como o constatado no presente trabalho.

\section{CONCLUSÕES}

A utilização do azeite em testes de larvas com papel impregnado é dispensável, não ocorrendo o mesmo nos testes de imersão de fêmeas ingurgitadas com compostos hidrofílicos. A mortalidade média causada por solventes é menor nos testes com papel impregnado, aumentando nos testes de imersão de larvas e de fêmeas. As fêmeas ingurgitadas são mais sensíveis aos solventes do que as larvas nos testes de imersão. Solventes de baixo peso molecular e pouca viscosidade não interferem na mortalidade média em testes biológicos de B. microplus, principalmente quando utilizados em concentrações inferiores a $76 \%$. Com os resultados obtidos, espera-se facilitar futuros trabalhos nos quais se busque a utilização de produtos naturais no controle do carrapato, como alternativa aos atuais produtos comerciais sintéticos.

\section{AGRADECIMENTOS}

Ao pesquisador da Embrapa Gado de Leite, Dr. Rui da Silva Verneque, pelo auxílio na análise estatística desse traba1ho. À FAPEMIG e à CAPES pelo importante auxílio através de bolsas de estudo.

\section{REFERÊNCIAS BIBLIOGRÁFICAS}

ABBOTT, W. S. A method for computing the effectiviness of insecticidies. J Econ Entomol, v.18, p.265-267, 1925.

BALASHOV, Y.S. A translation of bloodsucking ticks (Ixodoidea) - Vectors of diseases of man and animals. Misc Publ Entomol, v.8, n.5, p.159-376, 1972.

BEADLES, M.L.; DRUMMOND, R.O.; WHETSTONE, T.M. Tropical horse tick: effects of solvents on oviposition. J Econ Entomol, v. 66, p. 125-127, 1973.

CHUNGSAMARNYART, N,; JANSAWAN, W. Acaricidal activity of peel oil of Citrus spp. on Boophilus microplus. Kasetsart J (Nat Sci), v.30, p.112-117, 1996.

CHUNGSAMARNYART, N.; JIWAJINDA, S. Acaricidal activity of volatile oil from lemon and citronella grasses on tropical cattle ticks. Kasetsart J (Nat Sci), v.26, p.46-51, 1992.

CONSOLI, R.G.B. et al. Influência de diversos derivados de vegetais na sobrevida das larvas de Aedes fluviatilis (lutz) (Diptera: Culicidae) em laboratório. Mem Inst Oswaldo Cruz, v.83, n.1, p.87-93, 1988.

DRUMMOND, R. O. et al. Boophilus annulatus and $B$. 
microplus: laboratory tests of insecticides. J Econ Entomol, v.66, n.1, p.130-133, 1973

FAO PLANT PROTECTION BULLETIN. Recommended methods for the detection and measurement of resistance of agricultural pests to pesticides. Tentative methods for larvae of cattle tick Boophilus spp. FAO method n. ${ }^{0}$ 7, v.19, p.15-18, 1971 .

GEROLT, P. The mode of entry of contact insecticides. Pestic Sci, v.1, p.209-212, 1970.

GONZALES, J.C. O controle do carrapato dos bovinos. Porto Alegre : Sulina, 1974. 103p.

HADAWAY, A.B.; BARLOW, F. Some aspects of the effect of the solvent on the toxicity of solutions of insecticide. Ann Appl Biol, v.46, n.2, p.133-148, 1958.

HARRISON, R.A. Topical application of insecticide solutions to mites and small insectes. NZJ Sci, v.4, p.534-539, 1961.

HERNÁNDEZ, L. E.; PARRA, D. G.; MARIN, A. C. Accion repelente y acaricida del Melinis minutiflora sobre el Boophilus microplus. Rev Colombiana de Ciencias Químico Farmacéuticas, v.16, p.17-21, 1987.

KARR, L.L.; COATS, J.R. Insecticidal properties of $\alpha$ Limonene. J Pestic Sci, v.13, p.287-290, 1988.

LEITE, R. C. Boophilus microplus (Canestrini, 1887): susceptibilidade, uso atual e retrospectivo de carrapaticidas em propriedades das regiões fisiogeográficas da baixada do Grande-Rio e Rio de Janeiro. Uma abordagem epidemiológica. 1988. 151f. Tese (Doutorado em Medicina Veterinária Preventiva) - Curso de Pós-graduação em Medicina Veterinária, Universidade Federal de Minas Gerais.

ODHIAMBO, T.R. Current themes in tropical science: physiology of ticks. Oxford : Pergamon, 1982. V.1, 508p.

PASSOS, W.M. Contribuição para a melhoria no contro- le de algumas espécies de Malófagos (Insecta: Pitiiaptera), parasitos de galinhas de postura. 1994. 83f. Dissertação (Mestrado em Medicina Veterinária) - Curso de Pós-graduação em Parasitologia Veterinária, Universidade Federal Rural do Rio de Janeiro.

PORTER, R.B.R.; REESE, P.B.; WILLIAMS L.A.D. Acaricidal and inseticticidal activities of Candina-4,10(15)-dien-3-one. Phytochemistry, v.40, n.3, p.735-738, 1995.

REED, D.K.; CRITTENDEN, C.R.; LYON, D.J. Effect of ethyl and acetone on the toxicity of ethion in bioassays on two species of Rust Mites of Citrus. J Econ Entomol, v.61, n.4, p.1003-1005, 1968.

SAS - Statistics Analysis System. Versão 6. Cary, USA : SAS Institute, 1990. 1042p.

SHAW, R.D. Culture of an organophosphorus-resistant strain of Boophilus microplus (Can.) and an assessment of its resistance spectrum. Bull Ent Res, v.56, p.389-405, 1966.

SONENSHINE, D.E. Biology of ticks. Oxford : University Press, 1991. V.1, 447p.

SUJUTHA, C.H. et al. Evaluation of plant extracts for biological activity against mosquitoes. Int Pest Control, v.30, p.122-124, 1988.

THIELE, G.F.; HARRISON, R.A. Selection of solvents for topical application acaricide testing. Ent Exp Appl, v.11, p.389-396, 1968.

THULLNER, F. Impact of pesticide resistance and network for global pesticide resistance management based on a regional structure. World Anim Review, R M Z, v.89, p.41-47, 1997.

WILLIAMS, L.A.D. Adverse effects of extracts of Artocarpus altilis Park. and Azadirachta indica (A. Juss) on the reproductive physiology of the adult female tick, Boophilus microplus (Canest.). Invert Reprod Development, v.23, n.2-3, p.159164, 1993. 